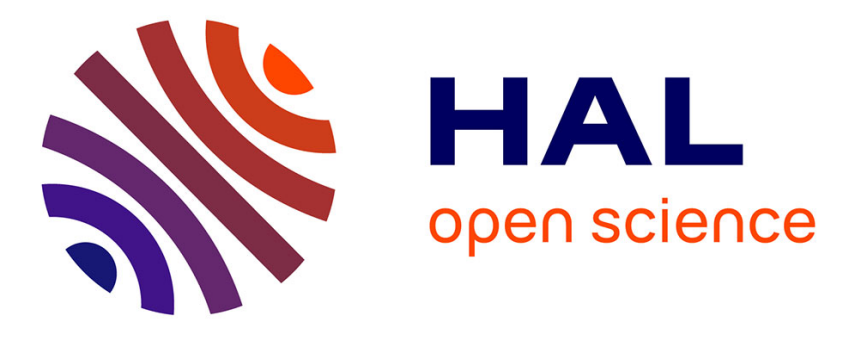

\title{
General Integral Formulation for the 3D Thin Shell Modeling
}

Tung Le Duc, Gérard Meunier, Olivier Chadebec, Jean-Michel Guichon, Joao Pedro A. Bastos

\section{- To cite this version:}

Tung Le Duc, Gérard Meunier, Olivier Chadebec, Jean-Michel Guichon, Joao Pedro A. Bastos. General Integral Formulation for the 3D Thin Shell Modeling. IEEE Transactions on Magnetics, 2013, 49 (5), pp.1989-1992. 10.1109/TMAG.2013.2242438 . hal-00821826

\section{HAL Id: hal-00821826 https://hal.science/hal-00821826}

Submitted on 13 May 2013

HAL is a multi-disciplinary open access archive for the deposit and dissemination of scientific research documents, whether they are published or not. The documents may come from teaching and research institutions in France or abroad, or from public or private research centers.
L'archive ouverte pluridisciplinaire HAL, est destinée au dépôt et à la diffusion de documents scientifiques de niveau recherche, publiés ou non, émanant des établissements d'enseignement et de recherche français ou étrangers, des laboratoires publics ou privés. 


\title{
General Integral Formulation for the 3D Thin Shell Modeling
}

\author{
Tung Le-Duc ${ }^{1}$, Gérard Meunier ${ }^{1}$, Olivier Chadebec ${ }^{1,2}$, Jean-Michel Guichon ${ }^{1}$, and Joao Pedro A. Bastos ${ }^{2}$ \\ ${ }^{1}$ Grenoble Electrical Engineering Laboratory, University of Grenoble, Grenoble INP/UJF/CNRS UMR 5269, Grenoble, France \\ ${ }^{2}$ GRUCAD/EEL/CTC/UFSC, Florianópolis, SC 88040-900, Brazil
}

In order to compute electromagnetic problems in presence of thin conductive and magnetic shells, a new integral formulation is proposed. Based on the principle of shell element, the formulation is general and enables the modeling of various problems for any skin-depth, avoiding the air region meshing. The formulation is validated thanks to an axisymmetric FEM and compared with another shell formulation implemented in a 3D FEM. Advantages and drawbacks of this new formulation are discussed.

Index Terms - Eddy currents, integral equation method, quasi-static, skin effect, thin shell.

\section{INTRODUCTION}

$\mathbf{T}$ HIN conductive magnetic shells are frequent in electromagnetic devices (such as planar conductors, electromagnetic shielding...) and the analysis of their eddy-current distributions is a complex problem in engineering.

The geometry of a thin region is characterized by a high ratio between length and thickness. Thus, using a volume mesh for the thin region leads to a large number of elements. Furthermore, when the frequency is high the skin depth becomes much thinner than the thickness $e$. It increases the size of the needed mesh if this effect is properly modeled. Shell element formulations have been developed to overcome such difficulties, e.g., with the boundary element method (BEM) [1], with the finite-element method [2]-[8] and with an integral method recently proposed by the authors of this paper [9].

In [9], a general shell element formulation for thin conductive regions modeling has been proposed $(\delta \gg e$ or $\delta \approx e$ or $\delta \ll e$ ). As in [1]-[8], this formulation takes into account the field variation through the depth due to the skin effect. The shell is modeled by an integral formulation avoiding the air region meshing. Based on a simple surface discretization of the shell averaged surface, the number of unknowns is considerably reduced, since its depth is not meshed. However, this already proposed shell element formulation was only suitable for conductive materials and could not be applied to shells with both magnetic and conductive properties.

This paper presents a new integral formulation which allows the modeling of magnetic and conductive thin shells in the general case $(\delta \gg e$ or $\delta \approx e$ or $\delta \ll e)$. This method is a natural extension of the method proposed in [9]. Moreover, we will see that an additional advantage is its implementation simplicity avoiding the complex computation of volume singular integrals as in [9]. Let use notice that the formulation is currently limited to linear cases but this assumption is often reliable for shielding effects computations.

In the second part of the paper, the integral equation governing the thin shell regions is presented. In the third part, this

Manuscript received October 29, 2012; revised January 03, 2013; accepted January 20, 2013. Date of current version May 07, 2013. Corresponding author: O. Chadebec (e-mail: Olivier.Chadebec@g2elab.grenoble-inp.fr).

Color versions of one or more of the figures in this paper are available online at $\mathrm{http}: / /$ ieeexplore.ieee.org.

Digital Object Identifier 10.1109/TMAG.2013.2242438

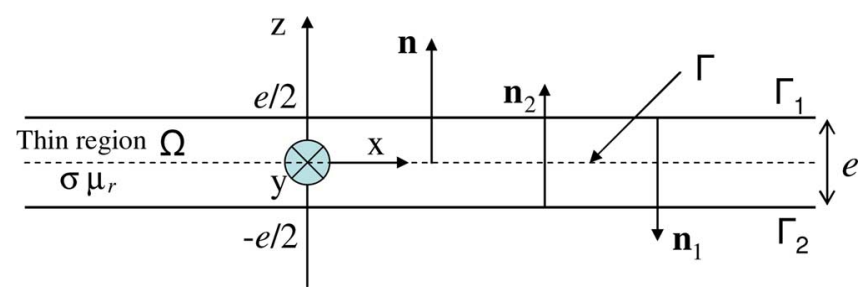

Fig. 1. Thin conductive and magnetic region and associated notations.

equation will be coupled with an integral formulation. Finally, a numerical example is proposed in the last part. Results obtained with our formulation are compared with results obtained with the Finite Element Method (FEM).

\section{THIN SHELL EQUATION}

Let $\Omega$ be a thin magnetic conductive shell with thickness $e$ (conductivity $\sigma$ and linear permeability $\mu_{\mathrm{r}}$ ) and $\Gamma_{1}, \Gamma_{2}$ boundaries of the shell with the air region (see Fig. 1.). We consider that a given skin depth $\delta$ is associated to the shell. The field variation of the tangential component across the thickness of shell can be approximated by the analytical solution of the problem for an infinite plane [1], [2]:

$$
\mathbf{H}_{s}(z)=\frac{1}{\operatorname{sh}(a e)}\left[\mathbf{H}_{1 s} \operatorname{sh}\left(\frac{a e}{2}+a z\right)+\mathbf{H}_{2 s} \operatorname{sh}\left(\frac{a e}{2}-a z\right)\right]
$$

where $a=(1+j) / \delta, \mathbf{H}_{1 s}$ and $\mathbf{H}_{2 s}$ are the tangential values of the magnetic field on both sides of the shell. Applying Galerkin's method on the Maxell-Faraday equation for the side "1" of the shell, we have [2], [9]:

$$
\int_{\Gamma_{1}} \operatorname{grad}_{\mathrm{s}} w \cdot\left(\alpha \mathbf{H}_{1 \mathrm{~s}}-\beta \mathbf{H}_{2 \mathrm{~s}}\right) \mathrm{d} \Gamma+\mathrm{j} \omega \int_{\Gamma_{1}} w \cdot \mathbf{B}_{1} \cdot \mathbf{n}_{1} \cdot \mathrm{d} \Gamma=0
$$

where $\alpha=a / \sigma t h(a e), \beta=a / \sigma s h(a e)$; w is a set of nodal surface weighting functions; $\mathbf{n}_{1}$ is the normal vector corresponding to the side " 1 " of the shell and $\lambda 1$ is the line region delimiting the $\Gamma 1$ surface.

The symmetrical equation corresponding to the other side of the shell is obtained by simply exchanging the indices " 1 " and "2":

$$
\int_{\Gamma_{2}} \operatorname{grad}_{\mathrm{s}} w \cdot\left(\alpha \mathbf{H}_{2 \mathrm{~s}}-\beta \mathbf{H}_{1 \mathrm{~s}}\right) \mathrm{d} \Gamma+\mathrm{j} \omega \int_{\Gamma_{2}} w \cdot \mathbf{B}_{2} \cdot \mathbf{n}_{2} \cdot \mathrm{d} \Gamma=0
$$


where $\mathbf{n}_{2}$ is the normal vector corresponding the side " 2 " of the shell and $\lambda_{2}$ is the line region delimiting the $\Gamma_{2}$ surface.

Equations (2) and (3) represent the electromagnetic behavior of the conductive shell itself. In order to take into account the surrounding air region, several authors choose the Finite Element Method [2]-[8]. Thus, the air region has to be also discretized.

In [9], we developed an integral approach based on the coupling of (2) and (3) with a volume integral equation. The obtained formulation presents an interesting compactness treating different air volumes as a single physical region.

However, this formulation still suffered from drawbacks. The first one is its application only on non magnetic materials. Another difficulty is the computation of volume integrals which can become very complicated to accurately consider the exponential decreasing of the current across the thickness, especially when the influence of an element on itself is focused. The following section proposes an approach for solving these issues.

\section{INTEGRAL FORMULATION}

Equations (2) and (3) are written on the averaged surface $\Gamma$ of the thin region. Subtracting (3) and (2) leads to:

$(\alpha+\beta) \int_{\Gamma} \operatorname{grad}_{\mathrm{s}} w\left(\mathbf{H}_{2 \mathrm{~s}}-\mathbf{H}_{1 \mathrm{~s}}\right) \mathrm{d} \Gamma+\mathrm{j} \omega \int_{\Gamma} w\left(2 \cdot \mathbf{B}_{\mathrm{a}} \cdot \mathbf{n}\right) \mathrm{d} \Gamma=0$

where $\mathbf{B}_{\mathrm{a}}=\left(\mathbf{B}_{1}+\mathbf{B}_{2}\right) / 2$ is the averaged induction and $\mathbf{n}$ is the normal vector corresponding to the surface $\Gamma$ (see. Fig. 1.).

A reduced magnetic scalar potential is now introduced on each side of the shell. Because of the jump of the magnetic field tangential component through the thickness, a double layer surface element is necessary [2]. The expressions related to the tangential magnetic fields on both sides and outside of the shell are:

$$
\mathbf{H}_{1 \mathrm{~s}}=\mathbf{H}_{01 \mathrm{~s}}-\operatorname{grad}_{\mathrm{s}} \phi_{1} \quad \mathbf{H}_{2 \mathrm{~s}}=\mathbf{H}_{02 \mathrm{~s}}-\operatorname{grad}_{\mathrm{s}} \phi_{2}
$$

where $\mathbf{H}_{01 \mathrm{~s}}$ and $\mathbf{H}_{02 \mathrm{~s}}$ are the tangential source fields generated by inductors at each side of the shell and $\phi_{1}, \phi_{2}$ are the both associated scalar reduced magnetic potentials.

Using (4) and (5) and assuming small variations of $\mathbf{H}_{0 \mathrm{~s}}$ through the shell, we get:

$$
(\alpha+\beta) \int_{\Gamma} \operatorname{grad}_{\mathrm{s}} w \cdot \operatorname{grad}_{s} \Delta \phi \mathrm{d} \Gamma+2 \mathrm{j} \omega \int_{\Gamma} w \cdot \mathbf{B}_{\mathrm{a}} \cdot \mathbf{n} \mathrm{d} \Gamma=0
$$

where $\Delta \phi=\phi_{1}-\phi_{2}$ is the scalar magnetic potential discontinuity, i.e. the potential jump of the double layer.

The magnetization law of the material is linear and associated to a constant relative permeability $\mu_{\mathrm{r}}$. The (6) can thus be rewritten as:

$$
\begin{aligned}
(\alpha+\beta) \int_{\Gamma} \operatorname{grad}_{\mathrm{s}} w & \operatorname{grad}_{s} \Delta \phi \mathrm{d} \Gamma \\
& +2 j \omega \frac{\mu_{0} \mu_{\mathrm{r}}}{\mu_{\mathrm{r}}-1} \int_{\Gamma} w \cdot \mathbf{M}_{\mathrm{a}} \cdot \mathbf{n} \mathrm{d} \Gamma=0
\end{aligned}
$$

where $\mathbf{M}_{\mathrm{a}}=\left(\mathbf{M}_{1}+\mathbf{M}_{2}\right) / 2$ is the averaged magnetization.

On the side " 1 " of the shell, the total magnetic field $\mathbf{H}_{1}$ is the sum of the inductor field $\mathbf{H}_{01}$, the field created by magnetization and the field created by eddy currents flowing in the shell. Hence an integral equation linking the local field to the magnetization and the eddy current can be written [10]:

$$
\frac{\mathbf{M}_{1}}{\mu_{\mathrm{r}}-1}=\mathbf{H}_{01}-\operatorname{grad} \frac{1}{4 \pi} \int_{\Omega} \frac{(\mathbf{M} \cdot \mathbf{r})}{\mathrm{r}^{3}} \mathrm{~d} \Omega+\frac{1}{4 \pi} \int_{\Omega} \frac{\mathbf{J} \times \mathbf{r}}{\mathrm{r}^{3}} \mathrm{~d} \Omega
$$

where $\mathbf{r}$ is the vector linking the integration point on $\Omega$ to the point where the field is expressed and $\mathbf{J}$ is the current density.

The integration of tangential magnetization through the depth can be expressed by [1], [2]:

$$
\int_{-e / 2}^{+e / 2} \mathbf{M d z}=\frac{\overline{\mathrm{G}}\left(\mathbf{M}_{1}+\mathbf{M}_{2}\right)}{2}=\overline{\mathbf{G}} \mathbf{M}_{\mathrm{a}}
$$

where $\overline{\mathrm{G}}=\operatorname{th}[(1+j) \mathrm{e} / 2 \delta] /[(1+j) \mathrm{e} / 2 \delta]$.

The equivalent shell current distribution $\mathbf{K}$ can be also expressed by [1], [2]:

$$
\mathbf{K}=\int_{-e / 2}^{+e / 2} \mathbf{J} d z=\mathbf{n} \times \operatorname{grad} \Delta \phi .
$$

Using (9) and (10), (8) becomes:

$$
\begin{aligned}
\frac{\mathbf{M}_{1}}{\mu_{\mathrm{r}}-1}=\mathbf{H}_{01}-\operatorname{grad} \frac{\overline{\mathrm{G}}}{4 \pi} \int_{\Gamma} \frac{\left(\mathbf{M}_{\mathrm{a}} \cdot \mathbf{r}\right)}{\mathrm{r}^{3}} \mathrm{~d} \Gamma \\
+\frac{1}{4 \pi} \int_{\Gamma} \frac{\mathbf{n} \times \operatorname{grad} \Delta \phi \times \mathbf{r}}{\mathrm{r}^{3}} \mathrm{~d} \Gamma
\end{aligned}
$$

The equation on the side " 2 " of shell is obtained by permuting the subscripts "1" and "2" as

$$
\begin{aligned}
\frac{\mathbf{M}_{2}}{\mu_{\mathrm{r}}-1}=\mathbf{H}_{02}-\operatorname{grad} \frac{\overline{\mathrm{G}}}{4 \pi} \int_{\Gamma} \frac{\left(\mathbf{M}_{\mathrm{a}} \cdot \mathbf{r}\right)}{\mathrm{r}^{3}} \mathrm{~d} \Gamma \\
+\frac{1}{4 \pi} \int_{\Gamma} \frac{\mathbf{n} \times \operatorname{grad} \Delta \phi \times \mathbf{r}}{\mathrm{r}^{3}} \mathrm{~d} \Gamma
\end{aligned}
$$

If we consider that $\mathbf{H}_{\mathbf{0}}$ has no variation through the thickness of the shell, we obtain:

$$
\begin{aligned}
\frac{\mathbf{M}_{\mathrm{a}}}{\mu_{\mathrm{r}}-1}=\mathbf{H}_{0}-\operatorname{grad} \frac{\overline{\mathrm{G}}}{4 \pi} \int_{\Gamma} \frac{\left(\mathbf{M}_{\mathrm{a}} \cdot \mathbf{r}\right)}{\mathrm{r}^{3}} \mathrm{~d} \Gamma \\
+\frac{1}{4 \pi} \int_{\Gamma} \frac{\mathbf{n} \times \operatorname{grad} \Delta \phi \times \mathbf{r}}{\mathrm{r}^{3}} \mathrm{~d} \Gamma
\end{aligned}
$$

Equations (7) and (13) have to be discretized. The easiest way is to mesh the averaged surface $\Gamma$, located halfway between both boundaries of the shells (see Fig. 1.), into $n$ triangular elements. They are associated to a uniform tangential component of the eddy current and uniform magnetization (meaning 1-order shape functions for the potential jump and 0 -order shape functions for the magnetization). The algebraic linear system obtained has $p+3 n$ complex unknowns (a single complex magnetization vector per element and a single complex magnetic scalar potential per node; the mesh being composed of $p$ nodes). Let 
us notice that shape functions with higher orders could be also used.

Equation (7) can be written with the following matrix form:

$$
[\mathbf{A}] \cdot[\Delta \Phi]+[\mathbf{B}] \cdot[\mathbf{M}]=0
$$

where:

$[\mathbf{M}]$ is a complex vector of dimension $3 n,[\Delta \Phi]$ is a complex vector of dimension $p$.

$[\mathbf{A}]$ is a $(p \times p)$ sparse matrix, expressed as follows:

$$
\mathrm{A}(\mathrm{i}, \mathrm{k})=(\alpha+\beta) \int_{\Gamma} \operatorname{grad}_{\mathrm{i}} \cdot \operatorname{grad}_{\mathrm{k}} \mathrm{d} \Gamma
$$

$[\mathbf{B}]$ is a $(p \times 3 n)$ sparse matrix and can be written as:

$$
\mathrm{B}(\mathrm{i}, \mathrm{k})=2 j \omega \frac{\mu_{0} \mu_{\mathrm{r}}}{\mu_{\mathrm{r}}-1} \int_{\Gamma} w_{\mathrm{i}} \cdot \mathbf{n}_{\mathrm{k}} \mathrm{d} \Gamma .
$$

A matrix system representing (13) can also be written thanks to a collocation approach or a most expensive Galerkin projection. Here a simple collocation at elements centroids can be used to get [10]:

$$
\left(\frac{\left[\mathbf{I}_{\mathrm{d}}\right]}{\left(\mu_{\mathrm{r}}-1\right)}+[\mathbf{F}]\right) \cdot[\mathbf{M}]-[\mathbf{C}] \cdot[\Delta \Phi]=\left[\mathbf{h}_{0}\right]
$$

where $\left[\mathbf{I}_{d}\right]$ represents the identity matrix; $\left[\mathbf{h}_{0}\right]$ is the source fields projected at element centroids, $[\mathbf{F}]$ is a $(3 n \times 3 n)$ dense matrix and $[\mathbf{C}]$ is a $(3 n \times p)$ also dense matrix. The coefficients of these matrices are expressed as follows:

$$
F(i, j)=\left\langle\left(\frac{\bar{G}}{4 \pi} \operatorname{grad} \int_{\Gamma_{j}} \frac{\mathbf{u}_{j} \cdot \mathbf{r}_{i}}{r_{i}^{3}} d \Gamma\right), \mathbf{u}_{i}\right\rangle
$$

where $\langle$,$\rangle denotes the classical scalar product operator and \mathbf{u}_{i}$ is the vector basis of cell element $i$ and $\mathbf{r}_{i}$ is the vector linking the integration point on $\Gamma_{j}$ to the centroid of element $i$.

$$
\mathrm{C}(\mathrm{i}, \mathrm{j})=\left\langle\left(\frac{1}{4 \pi} \int_{\Gamma_{\mathrm{j}}} \frac{\mathbf{n}_{\mathrm{j}} \times \operatorname{grad} \Delta \phi \times \mathbf{r}_{\mathrm{i}}}{\mathrm{r}_{\mathrm{i}}^{3}} \mathrm{~d} \Gamma\right), \mathbf{u}_{\mathrm{i}}\right\rangle
$$

Matrix terms (18) and (19) present singularities classically obtained with integral formulations so particular treatments are needed especially for the computation of the element influence on itself. Coefficients (18) are computed thanks an equivalent magnetic charge distribution located on edge of elements like proposed in [10]. The integral is computed numerically. This well-known approach avoids the superposition of Gauss points and points where the field has to be computed and thus suppress the singularity. For (19), we consider that the averaged internal field created by a conductor carrying a uniform current on itself is globally very low. Thus, the coefficient $\mathrm{C}(\mathrm{i}, \mathrm{i})$ is imposed to zeros while other coefficients $(i \neq j)$ are computed with standard numerical integration. Let us observe that in the proposed formulation, integrals are computed only thanks to a surface numerical integration. This is an important difference with [9] where accurate and uneasy volume integration across

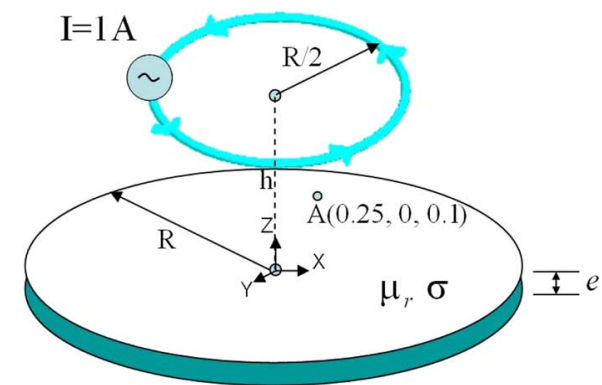

Fig. 2. Thin magnetic conductive disk $\left(\mathrm{R}=1 \mathrm{~m}, \mathrm{e}=50 \mathrm{E}-3 \mathrm{~m}, \mu_{\mathrm{r}}=100\right.$, $\sigma=6 \mathrm{E} 7 \mathrm{~S} / \mathrm{m}, \mathrm{h}=\mathrm{R} / 4)$.

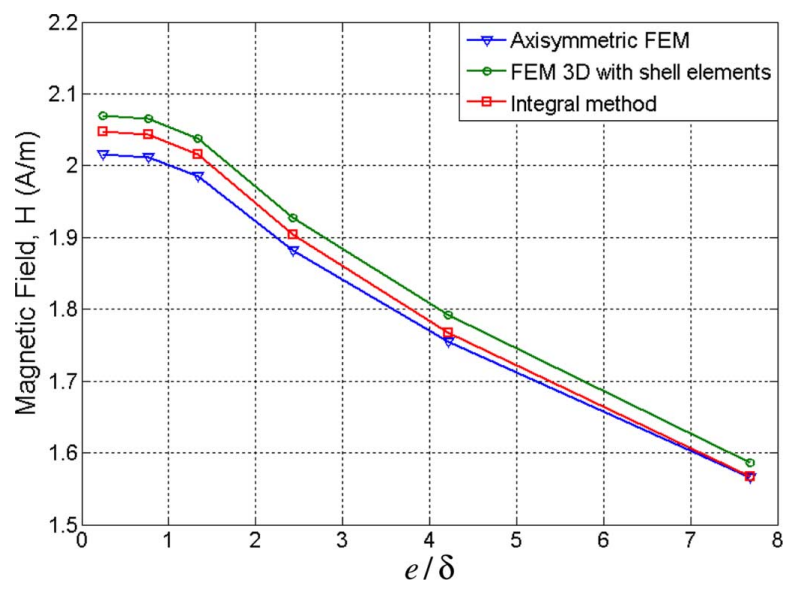

Fig. 3. Magnetic field in the point $\mathrm{A}(0.25,0,0.1)$, calculated by 3 methods versus $\mathrm{e} / \delta$.

the thickness was necessary. The method is then considerably simpler and reliable as shown in the following part.

\section{VALIDATION}

The formulation has been implemented for a 3D geometry and has been validated by two test problems. The first case is a thin magnetic conductive disk placed in a magnetic field $\mathbf{H}_{0}$ created by a current loop (Fig. 2.). This example is modeled by three different methods on a standard computer (Intel CPU $5160 @ 3 \mathrm{GHz} ; 16 \mathrm{~GB})$. The first one is the axisymmetric FEM using FLUX software [11] (100,000 elements, time of resolution $70 \mathrm{~s}$ ). The second one is a shell element formulation implemented in 3D FEM code [2], [7], [11] with 300,000 volume elements. The last one is the proposed integral method, the disk being meshed in 800 surface elements. We focus on the magnetic field computed in the air region for different frequencies at the point $\mathrm{A}(0.25,0,0.1)$ (Fig. 3$)$.

If we consider the axisymmetric FEM method as our reference, the integral method leads to an error of $1.6 \%$ for the magnetic field at $e / \delta=0.24$ and $0.2 \%$ at $e / \delta=7.7$ (Fig. 4). The integral formulation leads to more accurate results than the same implementation of the shell element but considering the air region treated with the 3D FEM. Let us notice that computation times are similar (around $300 \mathrm{~s}$ for 3D FEM and $240 \mathrm{~s}$ for the integral formulation).

The second test problem is the modeling of a real device proposed by EDF (Electricité de France) [12]. In order to limit short-circuit currents, the power station "Folies" is equipped 


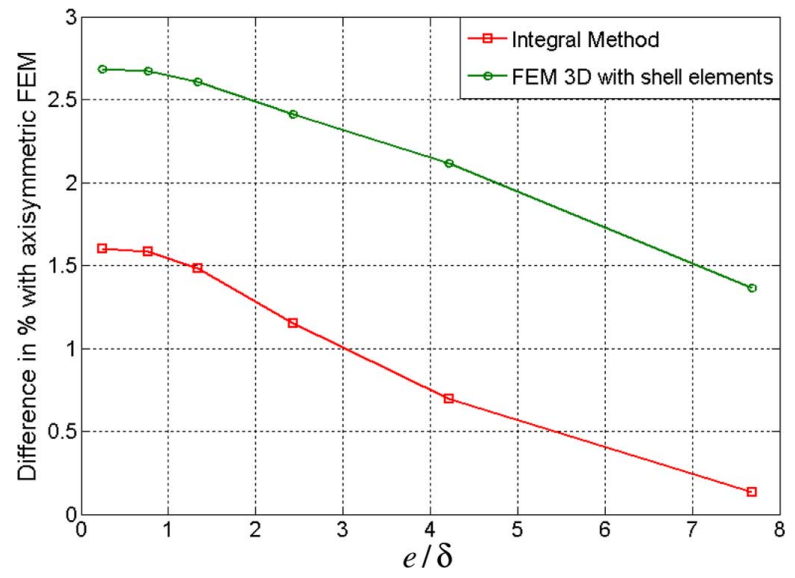

Fig. 4. Difference in $\%$ with the axisymmetric FEM solution for magnetic field computation versus $\mathrm{e} / \delta$.

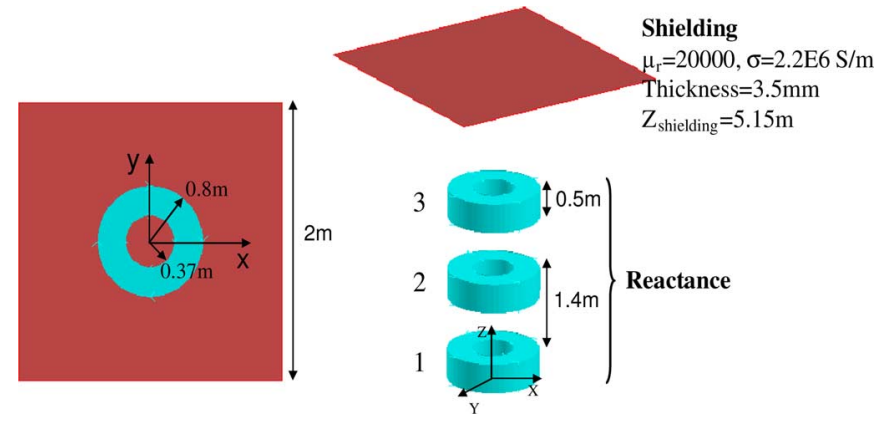

Fig. 5. Three reactances and electromagnetic shielding.

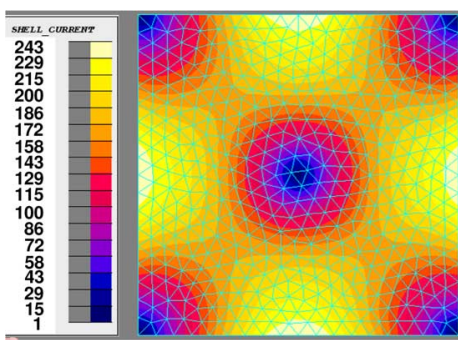

(a)

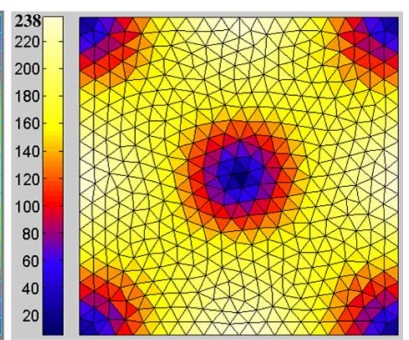

(b)
Fig. 6. Surface distribution of current $(\mathrm{A} / \mathrm{m})$ in the plaque for test case "Folies". a) FEM 3D with shell elements; b) Integral Method.

with three reactances in series with a power transformer secondary. The current in each reactance is 1000 A phase-shifted with 120 degree and the frequency is $50 \mathrm{~Hz}$. However, in nominal conditions, these three reactances create a leakage induction in the neighborhood. In order to minimize this electromagnetic disturbance, an electromagnetic shielding is added between the sources and the area to be protected. The skin depth $\delta=0.339 \mathrm{~mm}$ is smaller than the shielding thickness.

The Fig. 6 shows the current distribution computed by the proposed integral method and a more classical FEM 3D. Results provided by our coupling are very encouraging, the convergence being reached with few elements (less than 1000). This is the strong point of integral formulations which lead to accurate field computation in air regions with very low number of unknowns. However, some matrices obtained are fully populated and compression algorithms must be used if there are a large numbers of elements. In [13], [14], authors chose to use the well-known "Fast Multipole Method" (FMM) to compress matrices $[\mathbf{F}]$ and $[\mathbf{C}]$.

\section{CONCLUSiON}

In this paper, we have presented a new integral formulation using shell elements in order to model thin conductive and magnetic regions. The formulation is general and various skin depths $(\delta \gg e$ or $\delta \approx e$ or $\delta \ll e$ ) can be accurately modeled. It main advantage is its simplicity of implementation and its reliability. It drawbacks in comparison with our previous formulation proposed in [9] is that results are slightly less accurate for the field near the shell than in and we cannot compute the losses. However, the formulation remains quite efficient if problems where the magnetic field in the air and global shell current distribution must be evaluated. According to us, the coupling of this formulation with compression algorithms does not suffer from any difficulty.

\section{ACKNOWLEDGMENT}

This work was supported in part by the French National Research Agency (ANR/project E-CEM) and the Associated International Laboratory "James Clerk Maxwell” between France and Brazil (LIA 917-CNRS/CNPQ).

\section{REFERENCES}

[1] L. Krähenbühl and D. Muller, "Thin layers in electrical engineering. Example of shell models in analyzing eddy-currents by boundary and finite element methods," IEEE Trans. Magn., vol. 29, no. 2, pp. 1450-1455, 1993.

[2] C. Guérin, G. Tanneau, G. Meunier, P. Labie, T. Ngnegueu, and M. Sacotte, "A shell element for computing 3D eddy current-Application to transformers," IEEE Trans. Magn., vol. 31, no. 3, pp. 1360-1363, 1995.

[3] O. Bíró, A. Bárdi, K. Preis, W. Renhart, and K.-R. Richter, “A finite element formulation for eddy current carrying ferromagnetic thin sheets," IEEE Trans. Magn., vol. 33, no. 2, pp. 1173-1178, 1997.

[4] H. Igarashi, A. Kost, and T. Honma, "A three dimensional analysis of magnetic fields around a thin magnetic conductive layer using vector potential," IEEE Trans. Magn., vol. 34, no. 5, pp. 2539-2542, 1998.

[5] L. Krähenbühl, P. Dular, T. Zeidan, and F. Buretand, "Homogenization of lamination stacks in linear magnetodynamics," IEEE Trans. Magn., vol. 40, no. 2, pp. 912-915, 2004.

[6] V. Q. Dang, P. Dular, R. V. Sabariego, L. Krähenbühl, and C. Geuzaine, "Subproblem approach for thin shell dual finite element formulations," IEEE Trans. Magn., vol. 48, no. 2, pp. 407-410, 2012.

[7] C. Guérin and G. Meunier, "3-D magnetic scalar potential finite element formulation for conducting shells coupled with an external circuit," IEEE Trans. Magn., vol. 48, no. 2, pp. 323-326, 2012.

[8] P. Thomas and Y. Le Menach, "A three-dimensional electromagnetic shell finite element for coupled vector-scalar potential formulations," IEEE Trans. Magn., vol. 48, no. 2, pp. 823-826, 2012.

[9] T. Le-Duc, G. Meunier, O. Chadebec, and J.-M. Guichon, "A new integral formulation for eddy current computation in thin conductive shells," IEEE Trans. Magn., vol. 48, no. 2, pp. 427-430, 2012.

[10] O. Chadebec, J.-L. Coulomb, and F. Janet, "A review of magnetostatic moment method," IEEE Trans. Magn., vol. 42, no. 4, pp. 515-520, 2006.

[11] Flux Cedrat, Meylan, France [Online]. Available: www.cedrat.com

[12] A. Abakar, G. Meunier, J.-L. Coulomb, and F.-X. Zgainski, "3D modeling of shielding structures made by conductors and thin plates," IEEE Trans. Magn., vol. 36, no. 4, pp. 790-794, 2000.

[13] T.-S. Nguyen, J.-M. Guichon, O. Chadebec, P. Labie, and J.-L. Coulomb, "Ships magnetic anomaly computation with integral equation and fast multipole method," IEEE Trans. Magn., vol. 47, no. 5, pp. 1414-1417, 2011.

[14] A. Buchau, S. Tsafak, W. Hafla, and W. Rucker, "Parallelization of a fast multipole boundary element method with cluster OpenMP," IEEE Trans. Magn., vol. 44, no. 6, pp. 1338-1341, 2008. 